\title{
Noninvasive Means of Diagnosing Liver Fibrosis in Hepatitis C*
}

\author{
Eduardo Sellan Lopes Gonçales, Adriana Flávia Feltrim Angerami and Fernando Lopes Gonçales Junior \\ Study Group on Hepatitis, Infectious Diseases Division, UNICAMP; Campinas, SP
}

Liver biopsy is still considered the gold standard for staging fibrosis in chronic liver diseases. However, liver biopsy is an invasive procedure, and complications occur in $0.6 \%-5 \%$ of patients [1,2]. In addition, to perform the procedure there is a need for additional resources such as ultrasonography. Therefore, as a rule, patients undergoing liver biopsy are hospitalized for at least 6 hours [3].

Recent studies involving patients with chronic hepatitis $\mathrm{C}$ showed that fragments of technically inadequate hepatic tissue frequently lead to the underestimation of the stage of liver fibrosis [4]. That rate of diagnostic error can vary from 10\%-30\% depending on the study [5]. In addition, in developed countries, there is greater patient resistance to undergoing biopsy.

In Brazil, there is an additional factor, which is that patients are obligated to submit to liver biopsy for indication of treatment, except in clinically confirmed cases of hepatic cirrhosis according to the Ministry of Health guidelines.

For all of these reasons, an increasing number of studies are being conducted in order to evaluate the effectiveness of noninvasive markers for staging liver fibrosis. The noninvasive methods used in the largest number of published studies are the calculation of two indices - the aspartate aminotransferase (AST) to platelet ratio index (APRI) and the FibroTest index - and the FibroScan test.

The effectiveness of the various methods evaluated in various studies revealed quite heterogeneous results. The APRI method and the Forns index are unable to stage a large percentage of patients, and their accuracies do not exceed $80 \%-85 \%$. Therefore, a considerable number of patients are required to undergo liver biopsy. Otherwise, approximately $20 \%$ would be incorrectly diagnosed. The efficacy of those methods encounters difficulty regarding standardization and the definition of cut-off values for each degree of fibrosis.

\section{APRI}

The APRI was developed by Wai et al. [6] and is calculated based on AST levels and platelet counts. According to the results obtained in that study, the lower and upper cut-off values for the definition of significant fibrosis and cirrhosis are determined. Through analysis of the results, the positive and negative predictive values for the presence or absence of significant fibrosis or cirrhosis are also determined.

To evaluate significant fibrosis, the following cut-off values are used: lower than 0.5 (absence of significant fibrosis, Ishak stage 0-2); and higher than 1.5 (presence of significant fibrosis, Ishak stage 3-6) [6]. To evaluate cirrhosis, different cut-off values are used. The absence of cirrhosis (Ishak stage $0-4$ ) is defined as values lower than 1 , and cirrhosis (Ishak stage 5-6) is defined as values higher than 2 [6]. The formula for calculating the APRI test is as follows:

$$
A P R I=A S T(/ L S N) \times 100 / \text { Platelets }\left(10^{9} / \mathrm{L}\right)
$$

Table 1 shows the APRI values obtained.

Table 1. The aspartate aminotransferase to platelet ratio index results obtained by Wai et al.

\begin{tabular}{lccc}
\hline & Cut-Off & PPV & NPV \\
\hline No Fibrosis & $<0.5$ & $64 \%$ & $90 \%$ \\
Fibrosis & $>1.5$ & $91 \%$ & $65 \%$ \\
No Cirrhosis & $<1.0$ & $35 \%$ & $100 \%$ \\
Cirrhosis & $>2.0$ & $65 \%$ & $95 \%$ \\
\hline
\end{tabular}

$\mathrm{PPV}=$ positive predictive value; NPV=negative predictive value.

Therefore, the aforementioned Wai et al. showed that the APRI has a high positive predictive value to identify patients with significant fibrosis and a high negative predictive value to rule out cirrhosis. That study also showed that it is possible to predict the presence or absence of significant fibrosis in $51 \%$ of patients and to predict the presence or absence of cirrhosis in $81 \%$ of patients [6].

\section{FibroTest}

FibroTest combines and analyzes the serum levels of five factors in patients with chronic hepatitis $\mathrm{C}$. Those five factors are bilirubin, gamma-glutamyl transferase, apolipoprotein A1, alpha-2-macroglobulin and haptoglobin. The results obtained are evaluated through a formula which predicts and classifies them as F0-1, F2-3 and F4 [7].

\section{FibroScan - A New Noninvasive Method}

The evaluation of the degree of liver fibrosis is of fundamental importance to the prognosis, follow-up and therapeutic decision-making for patients with chronic liver disease. Biopsy is an invasive method and occasionally (although rarely) results in complications. In addition, the biopsy results, from an anatomical-pathological point of view, are often evaluated subjectively [8,9]. Nevertheless, biopsy continues to be the gold standard by which fibrosis is staged and evaluated

There are various studies on noninvasive options in the staging, evaluation and monitoring of liver fibrosis. FibroScan

* This article is part of the Proceedings of the Consensus of the Brazilian Society of Infectious Diseases on the Management and Treatment of Hepatitis C presented in the supplement of the Brazilian Journal of Infectious Diseases. 
Figure 1. Algorithm proposed by Sebastiani et al. (EASL) [14], in which the aspartate aminotransferase to platelet ratio index (APRI) method is used in conjunction with FibroTest.

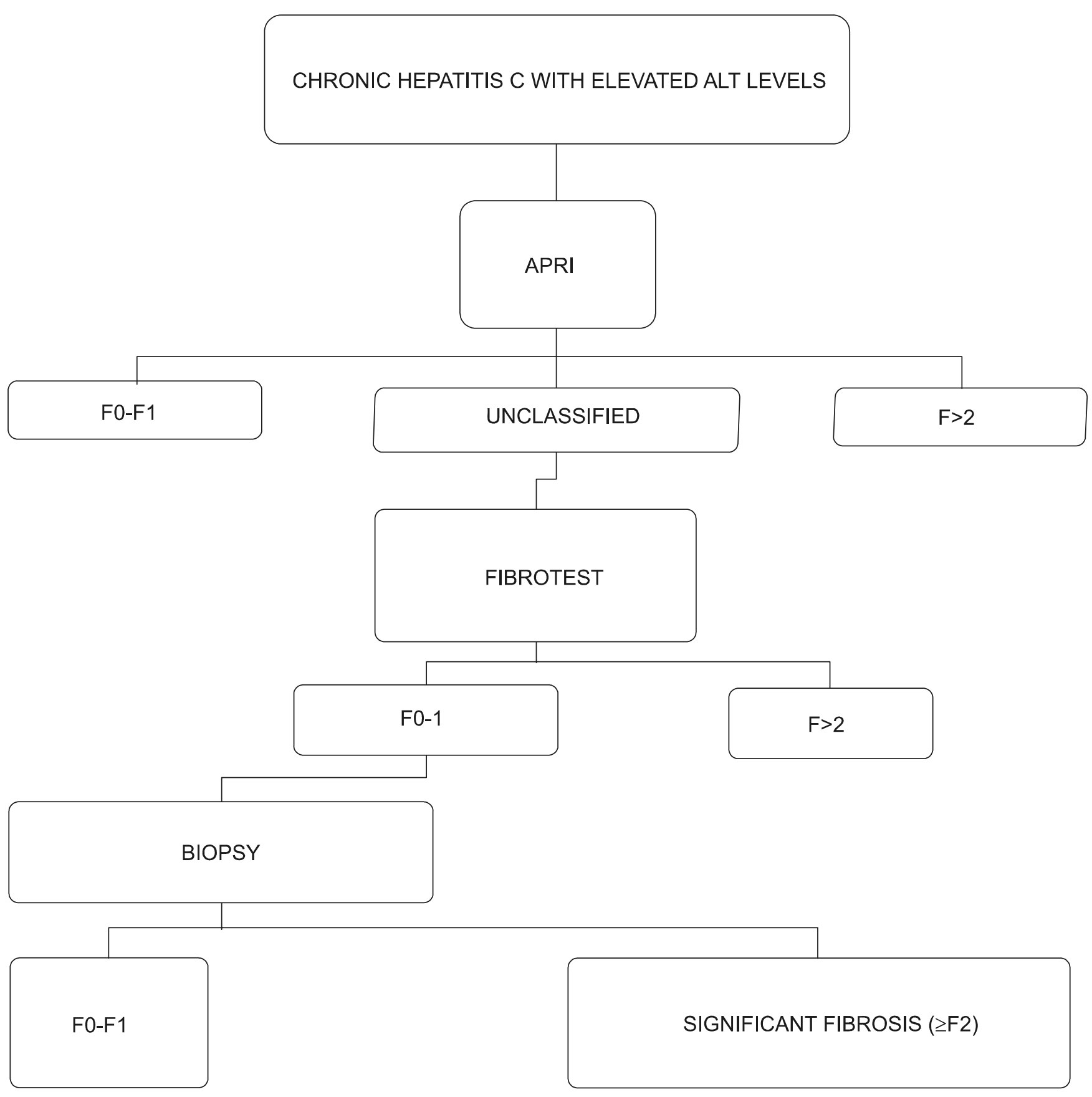

ALT=alanine aminotransferase; UDE=upper digestive tract endoscopy; US=ultrasound. 
Figure 2. Algorithm proposed by Castéra et al. [15], in which the FibroTest results are evaluated together with the FibroScan results.

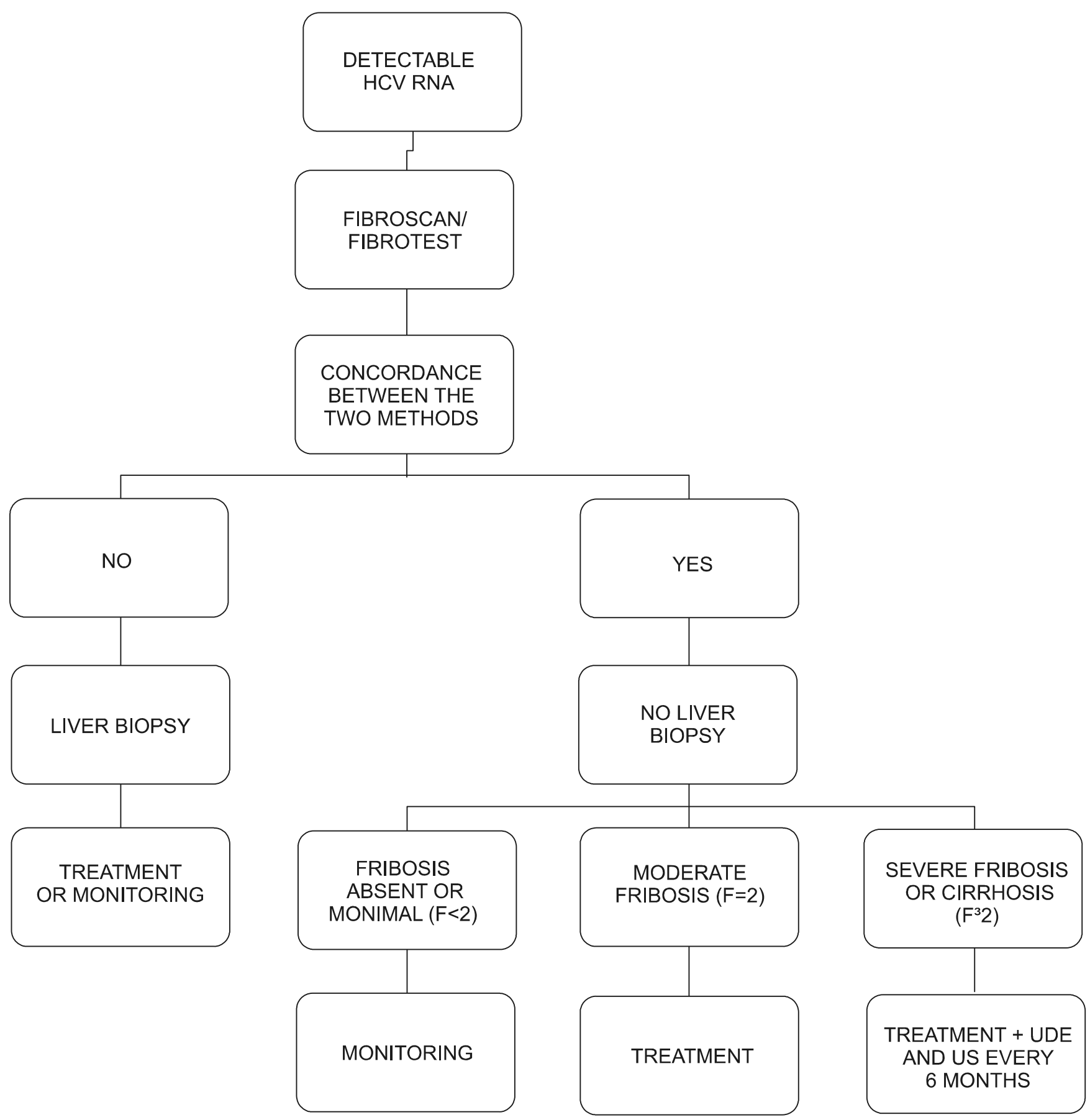


is a new method, still only available on a small scale, which presents better results in various studies with respect to differentiating between cirrhotic and noncirrhotic patients. It is considered a quick and easy noninvasive procedure for diagnosing cirrhosis and has been presented as an alternative to liver biopsy in patients with a formal contraindication [8].

FibroScan measures hepatic elasticity through a transducer positioned intercostally on the skin over the right lobe of the liver. The transducer transmits low amplitude and low frequency vibration pulses to the hepatic tissue. This vibration pulses propagate an elastic wave whose velocity is directly related to the elasticity of the tissue. Results are given in kilopascals $(\mathrm{kPa})$.

Some studies have shown that body mass index and age of the patient, as well as the level of experience on the part of the health professional, can influence the FibroScan results of patients with chronic hepatitis $\mathrm{C}$. The body mass index and steatosis can affect the evaluation of fibrosis, although some studies have shown that those factors are minimized if the test is repeated a fair number of times. Some studies recommend a total of five measurements to validate the results [10].

For the detection of fibrosis $\geq$ F2, FibroScan presents $85.2 \%$ sensitivity, $90.7 \%$ specificity, $93.8 \%$ positive predictive value, $78.8 \%$ negative predictive value and $87.7 \%$ diagnostic power. For the detection of cirrhosis, the test presents $78.3 \%$ sensitivity, $98.2 \%$ specificity, $97.8 \%$ positive predictive value, $81.6 \%$ negative predictive value and $88.2 \%$ diagnostic power [11-13].

Despite the reasonable quantity of published studies, few have compared the methods in a randomized manner. There are two studies that propose algorithms for evaluation. The first study, published by the European Association for the Study of the Liver (EASL), aims to standardize and compare the various methods based on serum markers of liver fibrosis. In its conclusion, the study proposes the use of an organogram that could reduce the need for liver biopsy by $60 \%-70 \%$ [14].

That study, conducted by Sebastiani et al. [14], evaluated the capacity of different methods to diagnose significant fibrosis (METAVIR fibrosis score $\geq 2$ ) in patients with normal or high levels of transaminase and to diagnose cirrhosis (Figure 1).

The results obtained show that significant fibrosis can be diagnosed with an accuracy of $94 \%$ using the APRI as the first screening test, followed by FibroTest in patients who were not classified through the APRI method, thereby limiting biopsy to only those patients in whom the degree of fibrosis is classified as F0-F1 using noninvasive methods [14].

Cirrhosis can also be diagnosed through this algorithm (95\% accuracy). The authors considered that in their original study they might have obtained highly favorable results due to the fact that the majority of patients presented significant fibrosis. The principal limitation of these markers is the difficulty in obtaining confirmation for patients with F0-F1 fibrosis. Therefore, an algorithm for the evaluation of fibrosis that attempts to define which patients are not required to undergo liver biopsy was developed.

Another study conducted by Castéra et al. [15] compared the effectiveness of transitory hepatic elastography (FibroScan, Echosens, Paris, France) in relation to the APRI and FibroTest. It succeeded in showing that FibroScan has a great capacity to diagnose significant fibrosis $(\geq F 2)$ (Figure 2). The results can be seen in Table 2 .

Table 2. FibroScan results

\begin{tabular}{lccc}
\hline \multirow{2}{*}{ Variable } & \multicolumn{3}{c}{ Degree of fibrosis } \\
\cline { 2 - 4 } & $\geq \mathrm{F} 2$ & $\geq \mathrm{F} 3$ & $\mathrm{~F} 4$ \\
\hline Sensitivity & $67 \%$ & $73 \%$ & $87 \%$ \\
Specificity & $89 \%$ & $91 \%$ & $91 \%$ \\
PPV & $95 \%$ & $87 \%$ & $77 \%$ \\
NPV & $48 \%$ & $81 \%$ & $95 \%$ \\
\hline
\end{tabular}

$\mathrm{PPV}=$ positive predictive value; $\mathrm{NPV}=$ negative predictive value

Therefore, it can be seen that FibroScan presents a high positive predictive value for patients with fibrosis $\geq F 2$ and an excellent negative predictive value for patients with hepatic cirrhosis. It is undoubtedly a good method for demonstrating significant fibrosis or for ruling out hepatic cirrhosis.

The study conducted by Castéra et al. proposes the combination of FibroScan and FibroTest as a screening method for significant fibrosis. When there was concordance between the methods, which occurred in $70 \%-80 \%$ of patients, the compatibility with the liver biopsy was $84 \%$ in diagnosing fibrosis $\geq F 2,95 \%$ in diagnosing fibrosis $\geq F 3$, and a $94 \%$ in diagnosing cirrhosis (F4).

Therefore, much still needs to be studied in relation to the noninvasive methods for estimating the degree of fibrosis, which is why it is necessary to carry out randomized comparative studies involving different patient populations.

\section{References}

1. Perrault J., McGill D.B., Ott B.j., Taylor W.F. Liver biopsy: complications in 1000 inpatients. Gastroenterology 1978; $74: 103-6$.

2. Gunneson T.J., Menon K.V., Wiesner R.H., et al. Ultrasoundassisted percutaneous liver biopsy perfomed by a physician assistant. Am J Gastroenterol 2002;97:1472-5.

3. Wong J.B., Koff R.S. Watchful waiting with periodic liver biopsy versus immediate empirical therapy for histologically mild chronic hepatitis C. A cost-effectiveness analysis. Ann Intern Med 2000; $133: 665-75$.

4. Colloredo G., Guido M., Sonzogni A., Leandro G. Impact of liver biopsy size on histological evaluation of chronic viral hepatitis: the smaller the sample, the milder the disease. J Hepatol 2003;39:239-44.

5. Poniachik J., Bernstein D.E., Reddy K.R., et al. The role of laparoscopy in the diagnosis of cirrhosis. Gastrointest Endosc 1996; $43: 568-571$

6. Wai C.T., Greenson J.K., Fontana R.J., et al. A simple noninvasive índex can predict both significant fibrosis and cirrhosis in patients with chronic hepatitis C. Hepatology 2003;38:518-26. 
7. Poynard T., McHutchison J., Manns M., et al. Biochemical surrogate markers of liver fibrosis and activity in a randomized trial of peginterferon alfa- $2 \mathrm{~b}$ and ribavirin. Hepatology 2003;38:481-92.

8. Transient elastography: a new surrogate marker of liver fibrosis influenced by major changes of transaminases. Journal of Viral Hepatitis 2007; 14:360-9.

9. Diagnosis of hepatic steatosis and fibrosis by transient elastography in asymptomatic healthy individuals: a prospective study of living related potencial liver donors. Journal of Gastroenterol 2007; $42: 382-8$.

10. Usefulneness of elastometry in evaluating the extents of liver fibrosis in hemophiliacs coinfected with hepatitis $\mathrm{C}$ and human immunodeficiency virus. Hepatology research 2006;35:135-9.
11. Features associated with success rate and performance of fibroscan measurements for the diagnosis of cirrhosis in HCV patients: a prospective study of 935 patients. Journal of Hepatology 2007; 46:628-34.

12. Transient elastography: a valid alternative to biopsy in patients with chronic liver disease. Journal compilation 2006;24:513-8.

13. Do not trivialize the Fibroscan examination, value its accuracy. Journal of Hepatology 2007;46.

14. Sebastiani G., Vario A., Guido M., et al. Stepwise combination algorithms of non-invasive markers to diagnose significant fibrosis in chronic hepatitis C. Journal of Hepatology 2006; 44:686-93.

15. Castéra L., Vergniol J., Foucher J., et al. Gastroenterology 2005; $128: 343-50$. 